\title{
EFFECT OF WATER AND SALINE LOAD ON URINARY OUTPUT IN HEALTHY UNDERGRADUATE MEDICAL STUDENTS
}

\author{
Paudel B H, Kumar S*
}

\section{ABSTRACT}

Water and electrolyte balance is a vital homeostatic function. Their excretion depends on various factors state of hydration, plasma osmolarity etc. There are rare reports on effect of hypertonic solution on urinary output. Therefore, the study aimed at investigating the effect of water or saline (especially the hypertonic) load on urinary output (UO) in healthy undergraduate male medical students (17-20 years). They (n=20) were randomised into 4 equal groups: control, water, normal and hypertonic saline (1.8\%). The study was a part of undergraduate practical conducted under controlled conditions in the Physiology Department, BPKIHS. The day before the experiment, all of them were given same instructions. Next day, the experimental groups drank water/saline solution $12 \mathrm{ml} / \mathrm{kg}$. Then the UO was measured 1/2-hourly. A non-parametric Friedman test was used for intra- and inter-group differences in UO and specific gravity. The data are presented as median (range). In water group the 60-min UO was significantly higher than the 30-min UO [178.5 (24-415) vs. $22.5(21-81) \mathrm{ml}, \mathrm{p}<0.05]$. This group also had significantly higher UO than the control at 90-min [84 (20-250) vs. $19(18-23) \mathrm{ml}, \mathrm{p}<0.05)]$. The UO of normal-saline group was significantly higher than that of control at 90-min [40 (22-250) vs. $19(18-23) \mathrm{ml}, \mathbf{p}<0.05]$. There was no significant difference in specific gravity. The water and normal saline loads were adequate to elicit physiological response, the hypertonic solution was not strong enough to show significant physiological response suggesting need of saline load to be more than twice of the plasma osmolarity.

\section{Key Words: homeostasis, water-electrolyte balance, salt load, water load}

\section{INTRODUCTION}

Water and electrolyte balance is a vital homeostatic function. Excretion of water in normal health is dependent on various physiological factors, e.g., state of hydration, state of solute load, plasma osmolarity, salt intake etc. Urinary output is altered in various pathological states and it is an important routine practice of measuring UO for monitoring condition of patients, especially of those who are critically ill. However, there are rare reports on effect of hypertonic solution on urinary output.

\section{OBJECTIVE}

The objective of the study was to investigate the effect of water and saline load, especially the hypertonic, on UO in healthy students. The study was a part of undergraduate practical conducted under controlled conditions in the department of Physiology, BP Koirala Institute of Health Sciences, Dharan, Nepal.

* Dept. of Physiology, B.P. Koirala Institute of Health Sciences, Dharan, Nepal.

Address for correspondence : $\quad$ Dr. Bishnu Hari Paudel

Assistant Professor, Dept. of Physiology

B.P. Koirala Institute of Health Sciences, Dharan, Nepal.

Email: bhpaudel@lycos.com 


\section{MATERIALS AND METHODS}

A total of 20 undergraduate students were recruited for the study. They were randomly divided into four groups: control, water load, normal saline solution $(0.9 \%)$ load, and hypertonic saline solution (1.8\%) load. Each group consisted of 5 students. The day before the experiment all the students were given the following instructions: no strenuous exercise, no consumption of alcoholic beverages, dinner at the same time, minimum of 6 hours of sleep, no smoking, and similar breakfast with only one glass of water on the day of experiment, appropriate clothing to keep oneself comfortable.

On the day of experiment all the students were asked to report to the Physiology Laboratory at 8 am. They were asked to void urine and measure their weight just before the experiment. Then they were given water or saline solution $12 \mathrm{ml} / \mathrm{kg}$ body weight to drink within 5 min. ${ }^{1}$ The control group did not take either water or saline solution. After drinking water (or saline) they were asked to sit comfortably and pass urine every half an hour. The amount of each UO (ml) sample and its specific gravity were measured every half an hour for $120 \mathrm{~min}$ thus obtaining four samples from each individual. Considering the sample size in each group and non-Gaussian distribution of the observations, a non-parametric (Friedman) test was used to compare the differences between or within the groups. The obtained data are presented as median and range. A $p$ value of less than 0.05 was considered as statistically significant.

\section{Table I : Study Design}

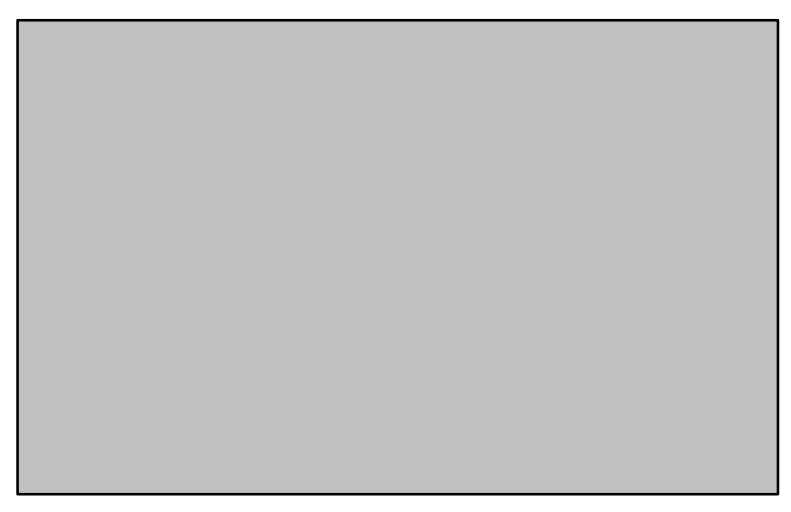

\section{RESULTS}

Urinary output and specific gravity of urine samples were compared between different 4 groups and within each group.

\section{Intra-group comparisons}

In the water group the $\mathrm{UO}$ at $60 \mathrm{~min}$ was significantly higher [178.5 (24-415) ml vs. $22.5(21-81) \mathrm{ml}, \mathrm{p}<0.05]$ than the output at 0.5 -hours of water load (Table II).
Table II : Urinary output of normal individuals

[median (range) ml]

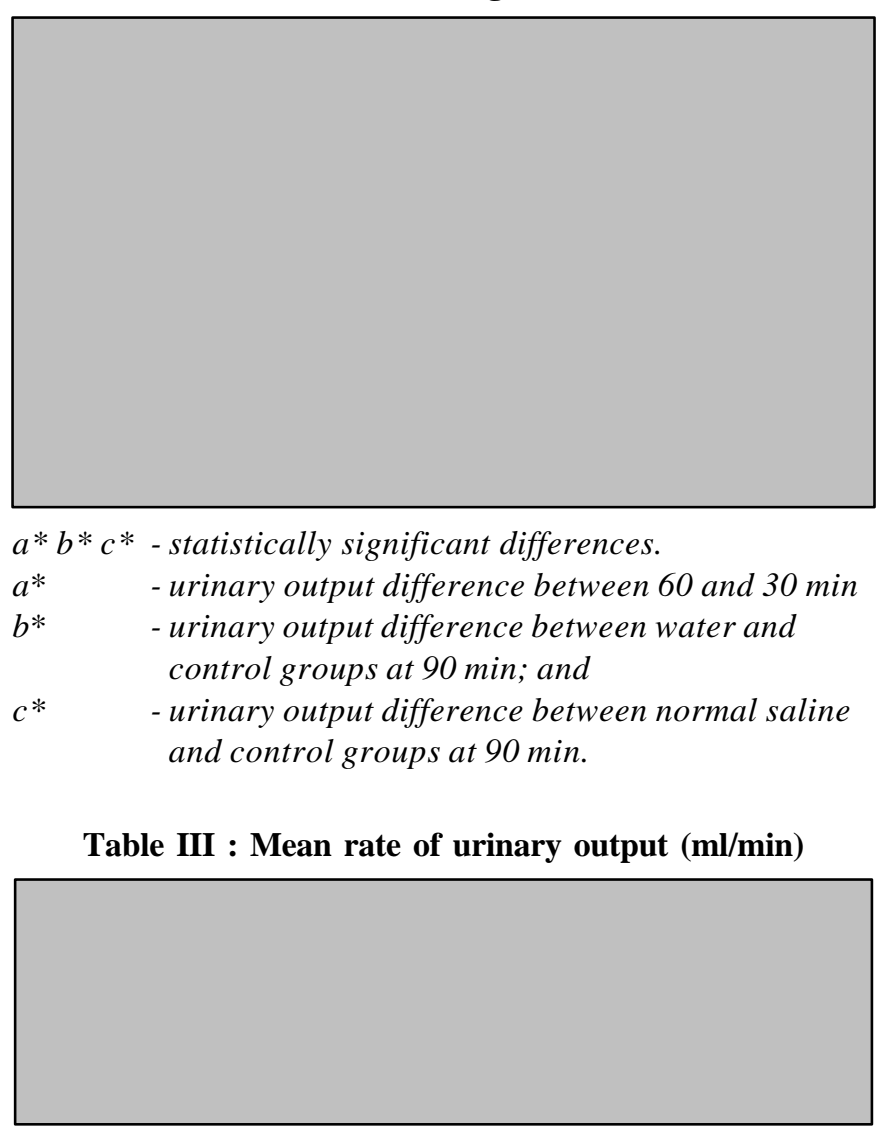

Table IV : Specific gravity of urine [median (range)]

\section{Inter-group comparisons}

The water group had significantly higher UO at $90 \mathrm{~min}$ than control group [84 (20-250) ml vs. 19 (18-23) ml, p<0.05]. Similarly the UO of normal saline group was also significantly higher [40 (22-250) ml vs. 19 (18-23) ml, p<0.05] than the output of control at $90 \mathrm{~min}$ of saline load (Table II). There were no statistically significant differences in specific gravity in any of between or within group comparisons (Table IV).

\section{DISCUSSION}

This study as a part of undergraduate practical of medical students was done under controlled conditions. The study aimed at investigating the effect of saline or water load on UO in healthy undergraduate students. The study showed some specific results. 
There was no effect of water and/or saline solution on diuresis and specific gravity at the initial $30 \mathrm{~min}$. However, the effect appeared at $60 \mathrm{~min}$ and $90 \mathrm{~min}$ maximally. It is known that osmo-receptor mechanisms are sensitive to solute load, therefore, there was no increment in UO in hypertonic saline load conserving water. ${ }^{2}$ Whereas in water and saline load groups there could have been decrease in antidiuretic hormone secretion allowing water loss by the kidneys. In both the situations extracellular osmolarity was maintained.

It has been hypothesised that the renal-fluid volume mechanism for control of the arterial pressure is an extremely powerful one, so powerful that it is either impossible or almost impossible for the arterial pressure ever to be adjusted to any other value besides the level dictated by this mechanism. ${ }^{3}$ Diuresis and natriuresis are known to occur in the absence of the adrenal glands and are independent from the neural tone that the adrenergic system exerts on sodium reabsorption. ${ }^{4}$ The diuretic response in the present study therefore might have occurred due to increase in blood volume leading to pressure diuresis. The release of atrial natriuretic peptide might have contributed to diuresis minimally since there was no significant change in specific gravity. Similarly, the role of antidiuretic hormone also seems minimal. Estimation of plasma osmolarity, which was not feasible in the study, would have given better insight into the mechanism.

The diuretic responses disappearing at $120 \mathrm{~min}$ in the present study suggests that $12 \mathrm{ml} / \mathrm{kg}$ body weight fluid load is not sufficient enough to maintain diuresis longer than 120 min. $^{2}$ The water or saline group as compared to hypertonic saline or control group exhibited a discernible increase in UO at 60 min (Tables II and III). In these groups, the increasing trend was sustained till the end of $2^{\text {nd }}$ hour of fluid load as compared to control group. The hypertonic saline group also had some trend towards increment in UO as compared to control group at $120 \mathrm{~min}$ of the load (Table II).

The effect of hypertonic saline was not seen being the weakest stimulus to evoke change in UO or its specific gravity. There was some trend towards increment of specific gravity in this group as compared to water and saline groups. This is in accordance with the known finding that retention of sodium induces reduction in water excretion without significant change in vasopressin, angiotensin II, aldosterone, or creatinine clearance. $^{5}$

In the present study, none of the stimuli given affected specific gravity significantly. However, there was a trend towards change when the water diuresis was high. The specific gravity of water or normal saline group showed decreasing trend as compared to the control group. The specific gravity of hypertonic saline group showed increasing trend as compared to water or normal saline group. Giving more water or highly concentrated $(>1.8 \%)$ saline solution to the individuals or follow-up for longer duration $(>2 \mathrm{~h}$ ) would have resulted into significant effect on measured variables especially the specific gravity. Fair constancy in specific gravity of urine indicates that the body primarily maintained the homeostasis through excretion of excess water although increase in UO is possible with hypertonic $\mathrm{NaCl}$ loading with sufficient extracellular fluid volume expansion. ${ }^{6}$ In the present study, hypertonic saline was the weakest among the stimuli, which could be because of unchanged glomerular filtration rate and renal plasma flow during Na loading. ${ }^{7}$

Self-control values would have given better comparison for individual groups. Nevertheless, the control group behaved very well showing uniform UO and specific gravity throughout the study period. We could not get the self-control values because of time constraints. In authors' opinion, the obtained data were sufficient enough for comparison and drawing some valid inference. High inter-individual variation in UO and specific gravity was seen, which could have been because of sample size in each group. Therefore, considering the nonGaussian distribution of observations a non-parametric test was, therefore, applied to see the difference between different comparisons.

The experiment was done during winter. Environmental and room temperatures were not measured since their effects were considered similar on all the participants of the study. If effect of exercise on UO has to be seen then the water or saline requirement would be higher. Previous study shows that it takes several days to compensate for a sodium loss caused by sweating with relatively low dose of salt intake. And it is recommended that extra salt be given during the exercise days. In the experimental situation, pre-exercise saline supplementation was found to be beneficial since the recovery time from exercise was shortened. ${ }^{8}$ Similarly if the experiment has to be done during summer season the amount of water and salt required inducing the obtained effect is likely to be more owing to greater loss of salt and water during summer through sweating. This type of studies can easily be designed and used for teaching of undergraduate and post-graduate medical students.

It is concluded that water was found to be the strongest stimulus to induce diuresis whereas hypertonic saline solution twice of the plasma tonicity could not induce any significant physiological effect. 


\section{REFERENCES}

1. Department of physiology. BP Koirala Institute of Health Sciences, Dharan, Nepal. Labex Manual for physiology. 19981999.

2. Guyton AC. Textbook of Medical Physiology. WB Saunders Company, USA, 1991, pp-313-315.

3. Guyton AC. Roles of the kidneys and fluid volumes in arterial pressure regulation and hypertension. ChinJ Physiol 1989; 32(2): 49-57.

4. Israel A, Torres M, Barbella Y. Natriuretic and diuretic action of centrally administered ratatrial natriuretic peptide (99-126): possible involvement of aldosterone and the sympatho-adrenal system. CanJ Physiol Pharmacol 1988; 66(3): 295-300.

5. Os I, Aakesson I, EngerE. Plasma vasopressin in hereditary cranial diabetes insipidus. Acta Med Scand 1985; 217(4): 429434.
6. Poujeol P, Chabardes D, Roinel N, De Rouffignac C. Influence of extracellularfluid volume expansion on magnesium, calcium and phosphate handling along the ratnephron. Pflugers Arch 1976; 365(2-3): 203-211.

7. Pedersen EB. Effectof sodiumloading and exercise on renal haemodynamics and uninary sodium excretion in young patients with essential hypertension before and during propranolol treatment. Acta Med Scand 1977; 201 (4): 365-373.

8. J ansson A, Nyman S, Morgan K, Palmgren-Karlsson C, LindholmA, Dahlborn K. The effectof ambienttemperature and saline loading on changes in plasma and urine electrolytes (Na and K) following exercise. Equine VetJ Suppl 1995; (20): 147-152.

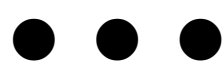

